\title{
Interplay between Classical Magnetic Moments and Superconductivity in Quantum One-Dimensional Conductors: Toward a Self-Sustained Topological Majorana Phase
}

\author{
Bernd Braunecker ${ }^{1}$ and Pascal Simon ${ }^{2}$ \\ ${ }^{1}$ Departamento de Física Teórica de la Materia Condensada, Centro de Investigación de Física de la Materia Condensada, \\ and Instituto Nicolás Cabrera, Universidad Autónoma de Madrid, E-28049 Madrid, Spain \\ ${ }^{2}$ Laboratoire de Physique des Solides, CNRS UMR-8502, Université Paris Sud, 91405 Orsay Cedex, France
}

(Received 11 July 2013; published 1 October 2013)

\begin{abstract}
We study a one-dimensional interacting electronic liquid coupled to a 1D array of classical magnetic moments and to a superconductor. We show that at low energy and temperature the magnetic moments and the electrons become strongly entangled and that a magnetic spiral structure emerges. For strong enough coupling between the electrons and magnetic moments, the 1D electronic liquid is driven into a topological superconducting phase supporting Majorana fermions without any fine-tuning of external parameters. Our analysis applies at low enough temperature to a quantum wire in proximity to a superconductor when the hyperfine interaction between electrons and nuclear spins is taken into account, or to a chain of magnetic adatoms adsorbed on a superconducting surface.
\end{abstract}

DOI: 10.1103/PhysRevLett.111.147202

PACS numbers: 71.10.Pm, 73.63.Nm, 74.45.+c, 75.75. - c

Introduction.-The interaction between localized magnetic moments and delocalized electrons contains the essential physics of many modern condensed matter systems. It is on the basis of nuclear magnets [1], heavy fermion materials of the Kondo-lattice type [2], or ferromagnetic semiconductors [3-6]. It often leads to new intricate physics and rich phases diagrams, even if the magnetic moments behave classically. Electron systems interacting with nuclear spins through the hyperfine interaction or magnetic adatoms with large magnetic moments arranged in some array on a metallic surface enter into this class.

In $1 \mathrm{D}$, the interactions between the nuclear spins and electrons lead to dramatic effects: below a crossover temperature $T^{*}$, a new exotic phase of matter in which the nuclear magnets are strongly tied to the electrons naturally emerges [7,8]. In this phase, the nuclear spins form a helical magnetic structure caused by the effective Ruderman-Kittel-Kasuya-Yosida (RKKY) interaction [9] mediated by the electron system. The feedback of this nuclear Overhauser field on the electron system entirely restructures the electronic states in that it opens a gap in one half of the elementary low-energy modes. The remaining electronic degrees of freedom remain gapless and form a quasihelical Luttinger liquid with strong analogies [10] with the edge states of the 2D quantum spin Hall effect $[11,12]$. Because of the mutual feedback, this order of strongly coupled electrons and nuclear spins is stable below a temperature $T^{*}$, and electron-electron interactions substantially enhance the stability [8]. Recent transport measurements in cleaved edge overgrowth GaAs quantum wires found a reduction of the conductance by a factor of 2 below $T<100 \mathrm{mK}$ independently of the density or applied magnetic field, consistent with this theory [13].
We stress that the mechanism behind this emergent helical structure is general, the essential ingredient being the RKKY interaction. Therefore, the same mechanism can apply if the nuclear spins are replaced by classical magnetic moments forming a 1D lattice (not necessarily a regular one), such as magnetic adatoms on top of a metallic surface [14].

When a finite-sized helical liquid is put in proximity of an $s$-wave superconductor, Majorana states can emerge at both ends [15] (see Fig. 1). This is the case for a quantum wire in the presence of spin-orbit coupling and a Zeeman term $[16,17]$ where some possible signatures of Majorana fermion physics have been recently reported experimentally [18-20]. The helical liquid, up to a gauge transformation [21], can also be obtained by coupling electrons to a spiral magnetic field (like the intrinsic nuclear Overhauser field [8]), or by manufacturing an external rotating magnetic field [22]. They can appear in rare-earth compounds exhibiting coexisting helical magnetism and superconductivity [23], or emerge by arranging magnetic adatoms in 1D arrays on the surface of a superconductor [24,25].

Since induced or intrinsic superconductivity entirely restructures the electron system, it is not a priori obvious

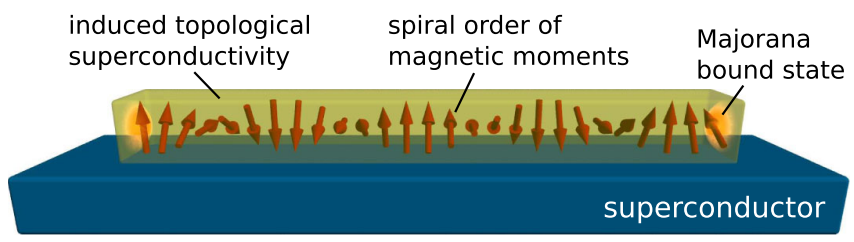

FIG. 1 (color online). A conductor with large magnetic moments placed on top of a superconductor. Topological superconductivity, Majorana bound states, and a spiral order of the magnetic moments emerge from a self-organization of the coupled systems of electrons and magnetic moments. 
whether the helical entangled states remain stable. In this work we provide such an investigation, carefully also taking into account electron-electron interactions and disorder. The underlying physics is the following: The RKKY interaction between the local moments has a strong $2 k_{F}$ component (with $k_{F}$ the Fermi wave vector), such that the local moments tend to be opposite at length $\lambda_{F} / 4$, with $\lambda_{F}=2 \pi / k_{F}$. The RKKY energy can thus be minimized if the local moments form a spiral $[7,8]$. This spiral acts back on the electron as the effective spiral Zeeman field required, together with the induced superconductivity, to drive the system into the topological superconductivity phase. Yet the opening of the superconducting gap as well as renormalizations by interactions and disorder again modify the RKKY interaction. Taking this self-consistently into account, however, we demonstrate that a selfstabilizing topological phase supporting Majorana bound states naturally emerges. We emphasize that this topological phase requires no fine-tuning. It is an intrinsic effect and constitutes the thermodynamic ground state of the system.

Model Hamiltonian.-We consider a 1D conduction electron liquid in the proximity of an $s$-wave superconductor. The 1D electrons are further coupled to an array of magnetic moments. Such a generic system is of the 1D Kondo-lattice type and described by the Hamiltonian

$$
H=H_{\mathrm{el}}+\sum_{x_{i}} A_{0} \mathbf{S}_{x_{i}} \cdot \mathbf{I}_{x_{i}} .
$$

The first term, $H_{\mathrm{el}}$, describes the electron system in the induced or intrinsic superconducting state and also includes electron-electron interactions. The second term describes the coupling between the electron spins $\mathbf{S}_{x_{i}}$ and the magnetic moments $\mathbf{I}_{x_{i}}$, weighted by a coupling constant $A_{0}$. The positions of the magnetic moments $x_{i}$ lie on a $1 \mathrm{D}$ chain. We assume that the distances between neighboring moments fulfill $\left|x_{i+1}-x_{i}\right| \ll \lambda_{F}$. Yet it is not required that the $x_{i}$ lie on a regular 1D lattice. The operators $\mathbf{S}_{x_{i}}$ are a tight binding representation of the electron spin operator in a region of dimension $\delta x \ll \lambda_{F}$ centered about $x_{i}$. The quantities $\mathbf{I}_{x_{i}}$ are either very large spins $I$ or composites of a large number $N_{\perp}$ of individual magnetic moments $I$ locked to a parallel configuration in some small volume at $x_{i}$ (such as nuclear spins in the transverse section of a quantum wire [8]). The former case is treated with $N_{\perp}=1$. We assume that $\mathbf{I}_{x_{i}}$ is normalized with respect to $N_{\perp}$ such that $A_{0}$ represents the interaction constant between an electron and one of the $N_{\perp}$ individual moments $I$ of $\mathbf{I}_{x_{i}}$. It has been shown numerically in [25] and further justified in [26] that this simple model captures qualitatively the behavior of a regular array of magnetic adatoms adsorbed on a superconducting surface (see also [27] for the $2 \mathrm{D}$ case).

The magnetic coupling $A_{0}$ also provides an effective RKKY [9] interaction and dynamics for the magnetic moments. This interaction is carried over the response of the electron system to magnetic perturbations and consists in an almost instantaneous long-ranged Heisenberg interaction between the magnetic moments $J\left(x_{i}-x_{j}\right)=$ $A_{0}^{2} a_{I}^{2} \chi\left(x_{i}-x_{j}\right) / 2$, where $\chi(x)$ is the electron spin susceptibility and $a_{I}$ the lattice spacing between the moments $I$. Note that generally $J$ can be anisotropic.

To analyze the Hamiltonian (1), we resort to a BornOppenheimer decoupling as in [8] which relies on the fact that the magnetic moments have a much slower dynamics than the electrons. Since the large moments $\mathbf{I}_{x_{i}}$ allow a (quasi)classical description, the terms $A_{0} \mathbf{I}_{x_{i}}$ act like a local quasistatic Zeeman field $\mathbf{B}_{\text {eff }}\left(x_{i}\right)=A_{0}\left\langle\mathbf{I}_{x_{i}}\right\rangle$ on the electron spins $\mathbf{S}_{x_{i}}$. The resulting effective theory is, therefore, expressed by the pair of Hamiltonians $[7,8,28]$

$$
\begin{gathered}
H_{\mathrm{el}}^{\mathrm{eff}}=H_{\mathrm{el}}+\sum_{x_{i}} \mathbf{B}_{\mathrm{eff}}\left(x_{i}\right) \cdot \mathbf{S}_{x_{i}}, \\
H_{m}^{\mathrm{eff}}=\sum_{x_{i}, x_{j}} J\left(x_{i}-x_{j}\right) \mathbf{I}_{x_{i}} \cdot \mathbf{I}_{x_{j}} .
\end{gathered}
$$

While $H_{\mathrm{el}}^{\text {eff }}$ acts only on electrons and $H_{m}^{\text {eff }}$ only on the magnetic moments, we stress that both Hamiltonians are strongly coupled since $\mathbf{B}_{\text {eff }}$ depends on the state of the magnetic moment system and $J$ on the electron state. A characterization of the physics described by Eqs. (2) and (3) must rely on a fully self-consistent approach, similar to the treatment of Refs. $[7,8,28]$.

Susceptibility in the noninteracting case.-Without superconductivity, the magnetic moments order in a spiral at low temperature due to the self-organization of the coupled systems [7,8], leading to an effective spiral field of amplitude $B_{\text {eff }}=A_{0} I$ and spatial period $\lambda_{F} / 2=\pi / k_{F}$. Since the spiral field rotates in a plane defining, for instance, the spin $(x, y)$ plane, the RKKY interaction is governed by the transverse spin susceptibility, defined as [8] $\chi^{\perp}(q)=-i \int_{0}^{\infty} d t \int d x e^{i q x}\left\langle\left[S_{+}(x, t), S_{-}(0,0)\right]\right\rangle$, for momenta $q$ with $S_{+}=\psi_{\uparrow}^{\dagger} \psi_{\downarrow}, S_{-}=S_{+}^{\dagger}$, and $\psi_{\sigma=\uparrow / \downarrow}(x)$ destroying an electron of spin $\sigma$ at position $x(\hbar=1$ throughout the Letter).

We first neglect electron interactions. Without superconductivity, $H_{\mathrm{el}}$ is then equivalent, by a gauge transformation [21], to a one-channel conducting wire with spin-orbit interaction in a magnetic field. The resulting band structure consists of two spin-mixing bands $\alpha= \pm$ with dispersions $E_{k}^{\alpha}=\epsilon_{k}+\alpha \sqrt{B_{\text {eff }}^{2}+v_{F}^{2} k^{2}}$, where $\epsilon_{k}$ is the single-particle dispersion and $v_{F}$ the Fermi velocity. At $k=0$ both bands are separated by a gap of amplitude $2 B_{\text {eff }}$, such that when the Fermi level lies within this gap, only band $\alpha=-$ remains conducting and forms a quasihelical conductor with roughly opposite spins at opposite Fermi points $k_{F-}$ (when undoing the gauge transformation $\left.k_{F-} \approx k_{F}\right)$. In the presence of superconductivity, the proximity induced gap $\Delta_{S}$ is expanded in the $\alpha= \pm$ eigenbasis and results in two triplet pairing terms within the " + " and 


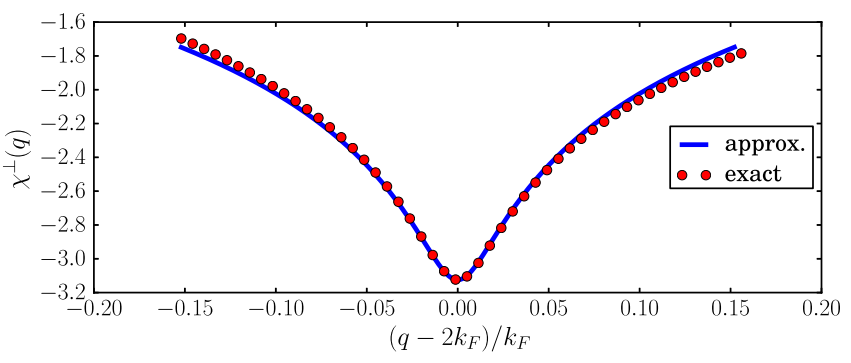

FIG. 2 (color online). Transverse susceptibility $\chi_{--}(q)$ for the noninteracting quasihelical conductor with induced superconductivity.

"_," bands, $\Delta=\Delta_{++}=\Delta_{--}$, and a singlet term $\Delta_{+-}$ that mixes both bands. A similar expansion holds for the transverse electronic susceptibility, which we write as $\left.\chi^{\perp}(q)=\chi_{--}^{\perp}(q)+\chi_{++}^{\perp} q\right)+\chi_{+-}^{\perp}(q)$. Since both bands are separated by a gap, $\chi^{\perp}(q)$ is dominated by the gapless "_" band such that $\chi^{\perp}(q) \approx \chi_{--}^{\perp}(q)$. The analytical form of $\chi_{-}^{\perp}(q)$ is derived in the Supplemental Material [29]. It has a deep minimum of the normal state susceptibility at $q=2 k_{F}$ (see Fig. 2). For $\Delta_{--} \ll v_{F} k_{F}$, we find (see Supplemental Material [29]) that it is well described by

$$
\chi_{--}^{\perp}(q) \approx \frac{-1}{2 \pi v_{F}}\left[\ln \left(\frac{2 v_{F} k_{F}}{\Delta}\right)-\frac{1}{\kappa} \operatorname{arctanh}(\kappa)\right],
$$

with $\quad \kappa=v_{F}\left(q-2 k_{F}\right) / \sqrt{4 \Delta^{2}+v_{F}^{2}\left(q-2 k_{F}\right)^{2}}$. Since $\chi \chi_{-}^{\perp}(q)$ maintains the deep minimum at $2 k_{F}$ characteristic of the normal state, this confirms on a mean-field level the consistency of the assumption of the spiral effective field, while stability under fluctuations will be considered below. We also note that the minimum can only be approximated by a Lorentzian of half-width $\Delta$ for $v_{F}\left|k-2 k_{F}\right| \ll \Delta$.

Susceptibility in the interacting case.-In $1 \mathrm{D}$ conductors, electron-electron interactions are often detrimental. To include them, we first linearize the spectrum in the absence of both the proximity induced gap and the spiral magnetic field. Our treatment is therefore valid only at low energy. We then use the standard approach of bosonizing the low-energy electronic Hamiltonian [30] and incorporate the pairing term and the spiral magnetic field in the bosonized Hamiltonian. The resulting low-energy model becomes $H_{\mathrm{el}}=H_{c}^{0}+H_{s}^{0}+H_{B}+H_{P}$, with

$$
\begin{gathered}
H_{\nu=c, s}^{0}=\int \frac{d x}{2 \pi}\left[\frac{v_{\nu}}{K_{\nu}}\left[\nabla \phi_{\nu}(r)\right]^{2}+v_{\nu} K_{\nu}\left[\nabla \theta_{\nu}(r)\right]^{2}\right], \\
H_{B}=\int \frac{d x}{2 \pi a} B_{\mathrm{eff}} \cos \left[\sqrt{2}\left(\phi_{c}+\theta_{s}\right)\right],
\end{gathered}
$$

and

$$
H_{P}=\int d x \frac{2}{\pi a} \Delta\left\{\sin \left[\sqrt{2}\left(\theta_{c}+\phi_{s}\right)\right]+\sin \left[\sqrt{2}\left(\theta_{c}-\phi_{s}\right)\right]\right\} .
$$

In Eq. (5), $K_{c, s}$ are the Luttinger liquid parameters for the charge and spin density fluctuations, $v_{c, s}$ is the charge and spin density wave velocities, and $a \sim a_{I}$ is a short distance cutoff. The noninteracting case is described by $K_{c}=K_{s}=1$ and $v_{c}=v_{s}=v_{F}$. Repulsive electronelectron interactions lead to $0<K_{c}<1$. If the spin SU (2) symmetry is preserved, $K_{s}=1$, otherwise $K_{s}>1$. Strictly speaking, this symmetry is broken here by $B_{\text {eff }}$, yet only weakly such that $K_{s} \approx 1$ [31,32]. The $\cos \sqrt{2}\left(\phi_{c}+\theta_{s}\right)$ term has a scaling dimension $\left(K_{c}+\right.$ $\left.K_{s}^{-1}\right) / 2$, and the $\cos \sqrt{2}\left(\theta_{c} \pm \phi_{s}\right)$ terms the scaling dimension $\left(K_{c}^{-1}+K_{s}\right) / 2$. In the noninteracting case both scaling dimensions are equal to 1 indicating the absence of any renormalization. For $1 / 3<K_{c}<1$ and $K_{s}=1$, both the pairing term and effective magnetic field terms are relevant; however, $B_{\text {eff }}$ dominates.

To make progress, we assume that $B_{\text {eff }} \gg \Delta$ (for strong repulsive interactions, one can relax this condition). This ensures that conduction modes unaffected by the opening of the $B_{\text {eff }}$ gap are helical, and that the induced superconductivity in this helical conductor is topological $[16,17,33]$. We proceed in a two-step renormalization group (RG) analysis. The coupling $B_{\text {eff }}$ reaches the strong coupling first, which opens a gap in the spectrum by pinning the field $\phi_{c}+\theta_{s}$ to a constant [8]. This physics is best accessed by introducing the fields $\left(\phi_{+}, \theta_{+}, \phi_{-}, \theta_{-}\right)$ related to the original fields by a unitary transform (see Supplemental Material [29]), with the \pm fields corresponding to the previous \pm bands of the noninteracting case. In this new basis, the effective Zeeman term gives a simple dominant sine-Gordon term $B_{\text {eff }} \cos \left(2 \phi_{+}\right)$. Hence, the gap opens only in the "+" sector, and from the strong coupling limit we can estimate it to be $B_{\text {eff }}^{*} \sim\left(v_{F} / a\right) \times$ $\left(a B_{\text {eff }} / v_{F}\right)^{2 /\left(4-K_{c}-K_{s}^{-1}\right)}$, coinciding with $B_{\text {eff }}$ in the noninteracting limit and increasing as a power law otherwise. After projecting out the gapped sector, the remaining Hamiltonian takes the simpler form

$$
H_{-}=H_{0}^{-}+\int d x \frac{2}{\pi a_{B}} \Delta^{\prime} \sin \left(2 \theta_{-}\right),
$$

where $H_{0}^{-}$is given by Eq. (5) with $K_{\nu}=K_{-}=2 K_{c} /(1+$ $\left.K_{c} K_{s}\right), v_{-}=v_{c} K_{s}^{-1}+v_{s} K_{c}$. Note that the effective bandwidth in this resulting " - " sector is now determined by $B_{\text {eff }}$ such that $a_{B} \sim v_{+} / B_{\text {eff }}$ replaces the UV cutoff $a$. The pairing term $\Delta$ is now replaced by the renormalized value $\Delta^{\prime} \lesssim \Delta$. The Hamiltonian (8) is just the bosonized version of a spinless electronic chain in the proximity of a superconductor. which can be studied by our second step RG procedure. As shown in Ref. [33], strong electron interactions further renormalize the value of $\Delta^{\prime}$, usually to $\Delta^{*} \ll \Delta^{\prime}$. However, by noticing that $K_{-}$also grows under the RG, we can refermionize the system at a length scale $\tilde{l}$ defined by $K_{-}(\tilde{l})=1$ [33]. The refermionized Hamiltonian is just a noninteracting 1D triplet superconductor with a renormalized pairing gap $\Delta^{*}$. The 
susceptibility is, therefore, given by Eq. (4) with the replacements $\Delta$ by $\Delta^{*} \ll \Delta$ and $v_{F}$ by $v_{-}$. Repulsive electron interactions therefore increase $B_{\text {eff }}^{*}$ while decreasing $\Delta^{*}$.

Stability analysis.-In the previous analysis, we have found that the RKKY interaction remains strongly peaked at $q=2 k_{F}$ in the presence of the superconductivity, such that the magnetic moments tend to form the spiral order. Through the opening of the gap in the electron system by this self-organization, this $2 k_{F}\left(\lambda_{F} / 2\right)$ spiral is strongly favored energetically. To investigate its stability, we perform a magnon analysis of $H_{m}^{\text {eff }}$ similar to Ref. [8], analyzing the low-energy fluctuations of the moments $\mathbf{I}_{x_{i}}$. If the $\mathbf{I}_{x_{i}}$ are regularly spaced and composed by $N_{\perp}$ individual moments of size $I$ that are ferromagnetically locked to each other, the magnetization $m$ per site (normalized to $0 \leq$ $m \leq 1) \operatorname{reads}[8] m=1-\left(1 / I N N_{\perp}\right) \sum_{q}\left(1 /\left(e^{\omega_{q} / k_{B} T}-1\right)\right)$, with $T$ the temperature, $k_{B}$ the Boltzmann constant, $N$ the number of sites of the chain, and the magnon dispersion $\omega_{q}=2 I\left(J_{q+2 k_{F}}-J_{2 k_{F}}\right) / N_{\perp}$, with $J_{q}=A_{0}^{2} a_{I} \chi_{--}(q)$. The order is stable as long as $m \approx 1$, and we denote the crossover temperature at which the order disappears by $T^{*}$. For an infinite chain, $T^{*}=0$ and order cannot be stable. For finite systems of length $L=N a_{I}$, this lowest mode is cut off, leading to $T^{*}>0$. Contrarily to the normal state case [8], the RKKY interaction $J_{q}$ is determined by $\Delta^{*}$ but not by $T$ (provided that $k_{B} T \ll \Delta^{*}$ ). Hence, $J_{q}$ remains invariant under temperature changes, and $T^{*}$ becomes a function of the remaining scales characterizing $\omega_{q}$, notably the cutoff scale $\omega_{2 \pi / L} \approx 2 I(2 \pi / L)^{2} d J_{q+2 k_{F}} /\left.d\left(q^{2}\right)\right|_{q=0}$, and the mean-field scale $\omega_{\infty}=2 I\left|J_{2 k_{F}}\right| / N_{\perp}$.

At very short system sizes such that only very few $q$ values fall into the dip of the RKKY interaction, we have $\omega_{q} \approx \omega_{\infty}$ for almost all terms in the $q$ summation, and we obtain $m \approx 1-\left[e^{\omega_{\infty} / k_{B} T}-1\right]^{-1} / I N_{\perp}$, which leads to a vanishing $m$ at temperatures exceeding

$$
k_{B} T^{*}=2 I^{2}\left|J_{2 k_{F}}\right|=A_{0}^{2} I^{2} a_{I}\left[\ln \left(2 v_{-} k_{F} / \Delta^{*}\right)-1\right] / \pi v_{-} .
$$

Quite remarkably, this result remains accurate up to large system lengths in which the approximation $\omega_{q} \approx \omega_{\infty}$ is no longer valid for most $q$. Indeed, let us consider the $L \rightarrow \infty$ limit, in which the sum to calculate $m$ is dominated by the $q=2 \pi / L$ term, $m \approx 1-\left[e^{\omega_{2 \pi / L} T}-1\right]^{-1} / I N N_{\perp}$. The associated temperature scale is $k_{B} T_{2 \pi / L}=I N \omega_{2 \pi / L} \sim$ $1 / L$. The length effects become influential only when $T^{*} \sim T_{2 \pi / L}$, i.e., at the length $L^{*} \approx \pi^{2} v_{-}^{2} /\left\{3 a_{I}\left(\Delta^{*}\right)^{2} \times\right.$ $\left.\left[\ln \left(2 v_{-} k_{F} / \Delta^{*}\right)-1\right]\right\} \sim\left(\xi^{*}\right)^{2} / a_{I}$. For systems with $L>$ $L^{*}$, the crossover temperature lies between $T^{*}$ and $T_{2 \pi / L}$, and decays with $L$ not faster than $T_{2 \pi / L} \sim 1 / L$. However, since usually $\xi^{*} / a_{I} \gg 1, T^{*}$ remains $L$ independent far into the regime $L \gg \xi^{*}$ required for obtaining isolated Majorana bound states. We notice that the unrenormalized
$I A_{0}$ in Eq. (9) must not be confused with the renormalized $B_{\text {eff }}^{*} \gg I A_{0}$ seen by the electrons. The topological phase requires $B_{\text {eff }}^{*}>\Delta^{*}$, yet within this situation both $I A_{0} \gg \Delta^{*}$ and $I A_{0} \ll \Delta^{*}$ are possible.

Disorder-We have assumed so far that the system is free of disorder. Since the RKKY magnetic interaction between the magnetic moments is mainly dominated by $J_{2 k_{F}}$, this remains the case even if the magnetic adatoms do not form a regular array. More problematic is the disorder in the electronic part. Semiconducting wires made out of GaAs or InAs are not free of disorder. A chain of adatoms on the surface also naturally introduces potential scattering terms. We introduce some quenched disorder $H_{\text {dis }}=$ $\int d x V(x) \rho(x)$ in $H_{\mathrm{el}}$, where $\rho(x)$ is the electron density and $V(x)$ encodes Gaussian disorder characterized by $\langle V(x) V(y)\rangle=D \delta(x-y)$, with $D$ the disorder strength. Technically, disorder introduces extra terms proportional to $\cos \sqrt{2}\left(\phi_{c} \pm \phi_{s}\right)$ in the bosonized Hamiltonian [30]. After disorder averaging, disorder terms generally compete with $B_{\text {eff }}$ and $\Delta$. As before, we assume again $B_{\text {eff }}$ to be larger than $\Delta$. By comparing the scaling dimensions of the disorder and spiral magnetic field terms [34], one finds that when $\left(D a / 4 \pi v^{2}\right)>(2 \Delta a / v)^{\gamma}$ with $\gamma=\left(3-K_{c}-\right.$ $\left.K_{s}\right) /\left(2-\left(K_{c}+K_{s}^{-1}\right) / 2\right) \quad\left(\right.$ we assumed $v=v_{c}=v_{s}$ here), disorder dominates and ultimately leads to localization. However, when $B_{\text {eff }}$ is the largest energy scale, we can project the disorder term into the "-" helical state which renders the disorder term, being nonmagnetic, inoperant at lowest order in $D[35,36]$. When all scales are of the same order, this is a difficult problem which goes beyond the scope of the present analysis. Therefore, the disorder energy scale must be at least smaller than the effective Zeeman field to observe the effect.

Application.-Let us first consider semiconducting GaAs or InAs wires. We take $L \sim 15 \mu \mathrm{m}, v_{F}=$ $2 \times 10^{5} \mathrm{~ms}^{-1}$, and $k_{F}=10^{8} \mathrm{~m}^{-1}$, and a proximity induced gap $\Delta_{S} \sim 0.2 \mathrm{meV}$ [18]. The hyperfine coupling for GaAs is $A_{0}=90 \mu \mathrm{eV}$ and $I=3 / 2$. For $K_{c}=1$, we obtain $B_{\text {eff }} \sim 0.2 \mathrm{meV}$ and $T^{*} \lesssim 1 \mathrm{mK}$, too small to be observed. More interesting is InAs, which has $I=9 / 2$ and $A_{0} \sim 110 \mu \mathrm{eV}$ [37]. For $K_{c}=0.8$, we obtain $T^{*} \sim 40 \mathrm{mK}$ and $B_{\text {eff }}^{*} \sim 0.5>\Delta^{*} \sim 0.1 \mathrm{meV}$ (which guaranties a topological SC phase), while for $K_{c}=0.6, \Delta^{*} \sim 200 \mathrm{mK}$ and $T^{*} \sim 70 \mathrm{mK}$, which is within experimental reach. For Co atoms on a $\mathrm{Nb}$ surface [25], we take $I=5 / 2, a_{I} \sim 3 \AA$, $E_{F}=v_{F} k_{F} / 2 \sim 10 \mathrm{meV}$, and $\Delta_{S} \sim 1 \mathrm{meV}$. For $K_{c}=1$, assuming a topological phase, i.e., $B_{\text {eff }} \sim I A_{0}>\Delta_{S}$, we find that $T^{*}>\Delta_{S} / k_{B}$. Therefore, a local magnetic coupling $A_{0}$ on the order of $0.5 \mathrm{meV}$, which is actually in the right range for magnetic exchange interactions [14], pushes the system in the topological phase.

Conclusion.-We have shown that in 1D a strong entanglement between magnetic moments and the electrons leads naturally at low temperature to a magnetic spiral structure. Combined with a proximity induced 
superconducting gap, this structure can drive the system into a topological superconducting phase supporting Majorana fermions. A fine-tuning of external parameters is not required. This scenario applies to semiconducting wires with nuclear spins or to a chain of magnetic adatoms on top of a superconductor surface. We also demonstrated that moderate electron interactions help stabilize the topological phase.

We thank M. Franz, D. Loss, and P. Wahl for helpful discussions and correspondences. P. S. would like to thank the department of physics at UBC (Vancouver) for its kind hospitality during the final stage of this work. B. B. acknowledges support by the EU-FP7 project SE2ND [271554].

Note added.-Recently, we became aware of Refs. [38,39] which has overlaps with the present work in the noninteracting limit.

[1] H. Fröhlich and F. R. N. Nabarro, Proc. R. Soc. A 175, 382 (1940).

[2] H. Tsunetsugu, M. Sigrist, and K. Ueda, Rev. Mod. Phys. 69, 809 (1997).

[3] H. Ohno, H. Munekata, T. Penney, S. von Molnár, and L. L. Chang, Phys. Rev. Lett. 68, 2664 (1992).

[4] H. Ohno, Science 281, 951 (1998).

[5] T. Dietl, A. Haury, and Y. M. d'Aubigné, Phys. Rev. B 55, R3347 (1997).

[6] J. König, H.-H. Lin, and A. H. MacDonald, Phys. Rev. Lett. 84, 5628 (2000).

[7] B. Braunecker, P. Simon, and D. Loss, Phys. Rev. Lett. 102, 116403 (2009).

[8] B. Braunecker, P. Simon, and D. Loss, Phys. Rev. B 80, 165119 (2009).

[9] C. Kittel, Quantum Theory of Solids (J. Wiley \& Sons, New York, 1987)

[10] B. Braunecker, C. Bena, and P. Simon, Phys. Rev. B 85, 035136 (2012).

[11] C. L. Kane and E. J. Mele, Phys. Rev. Lett. 95, 226801 (2005).

[12] M. König, S. Wiedmann, C. Brüne, A. Roth, H. Buhmann, L. W. Molenkamp, X.-L. Qi, and S.-C. Zhang, Science 318, 766 (2007).

[13] C.P. Scheller, T.-M. Liu, G. Barak, A. Yacoby, L. N. Pfeiffer, K. W. West, and D. M. Zumbühl, arXiv:1306.1940.

[14] M. Menzel, Y. Mokrousov, R. Wieser, J. E. Bickel, E. Vedmedenko, S. Blügel, S. Heinze, K. von Bergmann, A. Kubetzka, and R. Wiesendanger, Phys. Rev. Lett. 108, 197204 (2012).

[15] M. Z. Hasan and C. L. Kane, Rev. Mod. Phys. 82, 3045 (2010).
[16] R. M. Lutchyn, J. D. Sau, and S. Das Sarma, Phys. Rev. Lett. 105, 077001 (2010).

[17] Y. Oreg, G. Refael, and F. von Oppen, Phys. Rev. Lett. 105, 177002 (2010).

[18] V. Mourik, K. Zuo, S. M. Frolov, S. R. Plissard, E. P. A. M. Bakkers, and L.P. Kouwenhoven, Science 336, 1003 (2012).

[19] M. T. Deng, C. L. Yu, G. Y. Huang, M. Larsson, P. Caro, and H. Q. Xu, Nano Lett. 12, 6414 (2012).

[20] A. Das, Y. Ronen, Y. Most, Y. Oreg, M. Heiblum, and H. Shtrikman, Nat. Phys. 8, 887 (2012).

[21] B. Braunecker, G. I. Japaridze, J. Klinovaja, and D. Loss, Phys. Rev. B 82, 045127 (2010).

[22] M. Kjaergaard, K. Wölms, and K. Flensberg, Phys. Rev. B 85, 020503(R) (2012).

[23] I. Martin and A. Morpurgo, Phys. Rev. B 85, 144505 (2012).

[24] T.-P. Choy, J. M. Edge, A. R. Akhmerov, and C. W. J. Beenakker, Phys. Rev. B 84, 195442 (2011).

[25] S. Nadj-Perge, I. K. Drozdov, B. A. Bernevig, and A. Yazdani, Phys. Rev. B 88, 020407(R) (2013).

[26] F. Pientka, L. Glazman, and F. von Oppen, arXiv:1308.3969.

[27] S. Nakosai, Y. Tanaka, and N. Nagaosa, arXiv:1306.3686.

[28] P. Simon and D. Loss, Phys. Rev. Lett. 98, 156401 (2007); P. Simon, B. Braunecker, and D. Loss, Phys. Rev. B 77, 045108 (2008).

[29] See Supplemental Material at http://link.aps.org/ supplemental/10.1103/PhysRevLett.111.147202 for detailed calculations of the noninteracting susceptibility together with the bosonized form of the Hamiltonian and the unitary transform.

[30] T. Giamarchi, Quantum Physics in One Dimension (Oxford University Press, Oxford, 2004).

[31] J. Sun, S. Gangadharaiah, and O. A. Starykh, Phys. Rev. Lett. 98, 126408 (2007); S. Gangadharaiah, J. Sun, and O. A. Starykh, Phys. Rev. B 78, 054436 (2008).

[32] A. Schulz, A. De Martino, P. Ingenhoven, and R. Egger, Phys. Rev. B 79, 205432 (2009).

[33] S. Gangadharaiah, B. Braunecker, P. Simon, and D. Loss, Phys. Rev. Lett. 107, 036801 (2011).

[34] B. Braunecker, A. Ström, and G. I. Japaridze, Phys. Rev. B 87, 075151 (2013).

[35] X.-L. Qi and S. C. Zhang, Rev. Mod. Phys. 83, 1057 (2011).

[36] Here this becomes manisfest as terms $\propto D \cos \left(\kappa_{1} \phi_{-}\right) \times$ $\cos \left(\kappa_{2} \theta_{-}\right)$, which are of zero average, where $\kappa_{1,2}$ are unimportant functions of $K_{c}, K_{s}$.

[37] W. A. Coish and J. Baugh, Phys. Status Solidi B 246, 2203 (2009).

[38] J. Klinovaja, P. Stano, A. Yazdani, and D. Loss, arXiv:1307.1442.

[39] M. Vazifeh and M. Franz, arXiv:1307.2279. 